\title{
Small-Angle Scattering of Microparacrystallites (mPC's)
}

(Extended Abstract only) By R. HoSEMANN and J. LOBODA-ČAČ KOvić, Fritz-Haber-Institut der MPG, Teilinstitut für Strukturforschung, Berlin-Dahlem, Germany and K. KaJI, University of Kyoto, Department of Polymer Chemistry, Kyoto, Japan

(Received 3 November 1977; accepted 8 February 1978)

Two-dimensional small-angle X-ray scattering (SAXS) analysis combined with wide-angle X-ray scattering (WAXS) from highly oriented polymers proves that the polymers consist of mosaic blocks with paracrystalline distortions - the so-called microparacrystallites ( $\mathrm{MPC}$ 's), which are the bricks of a paracrystalline superlattice - the so-called macroparacrystal (MPC). The concept of meso-phases fails, because the MPC's show simultaneously both nematic and smectic properties. All this new information cannot be obtained with slit-smearing cameras and unoriented samples.

An interesting example is the SAXS four-point diagram of biaxially oriented polyethylene film (Kaji, Mochizuki. Akiyama \& Hosemann, 1978). It is impossible to explain it by the well-known two-phase model of 'crystalline' lamellae. One has to take into account statistical correlations between the surfaces of adjacent lamellae which influence the SAXS remarkably. The conventional explanation of fourpoint diagrams is given in the left-hand side of Fig. 1. A bundle of lamellae, tilted away from the stretching direction $x_{3}$ (vertical in the figure) by an angle $\varphi$ can most conveniently be described by the folding product of the shape function of one lamella with the one-dimensional point function of the positions of the centers of the lamellae. In Fourier space this is the product of the Fourier transform of one lamella (LS) with the Fourier transform EL of the lattice point function. This function is assumed for simplicity (at the bottom left of the figure) to be crystalline and to consist therefore of a series of equidistant thin discs. Two of the maxima of the observed four-point diagram (center of the figure) are thus explained. Near the other surface of the film the other corresponding families of tilted lamellae produce the other two maxima.

The conventional explanation fails, since

(1) the lateral sizes of the maxima are always much broader than expected from the lateral dimensions of the lamellae;

(2) the bundles always have quite irregular lateral boundaries and therefore cannot determine the direction of the flat maxima, which in our case are tilted by $\psi=12$ away from the normal to the stretching direction.

An adequate explanation is given on the right-hand side of the figure. The lamellae consist of mPC's whose centers build up a MPC with supercell edges $a_{3}$ and $a_{r}$. The vectors fluctuate statistically in length and direction from superlattice cell to superlattice cell and build up two families of discs in Fourier space each orthogonal to the respective mean edge vector. Unlike the thin crystalline discs, they are more and more broadened with increasing distance from the primary beam. Their product has two maxima, which explain quantitatively the shape of the SAXS maxima if one introduces adequate statistical fluctuations of $a_{3}$ and $a_{r}$. The maxima of the four-point diagram then have the Miller indices 01 and $0 \overline{1}$ of the superlattice, on which the shape of the bundle of lamellae has no influence. Multiplying the product of the discs with the shape factor of the MPC's, one obtains an intensity function which defines quantitatively the shape of the SAXS maxima. The intensity functions depend in their lateral direction on the lateral size of the mPC's and the relative statistical fluctuation of $a_{r}$ in the direction of $a_{3}$ called $g_{r 3}$. The two-dimensional analysis of the SAXS shows that for $g_{r 3}>0.5$ (nematic-like superstructures) the lateral shape of the maxima is given solely by the lateral size of the mPC's (Loboda-Čačković, Hosemann, Čačković, Ferrero \& Ferracini, 1976) and not by the size the bundle. With decreasing $g_{r 3}$ the influence of the paracrystalline superlattice factor become more pronounced. Hard elastic fibers with substantially smectic-like lamellae show SAXS reflections of small lateral dimensions corresponding to $1000 \AA$ or more (Noether \& Whitney, 1973), produced by a couple of mPC's well aligned in the lateral direction.

The concept of mesophases (Hermann, 1931a,b) fails, because it cannot describe structures with simultaneous nematic- and smetic-like (fibrillar and lamellar) properties. Using the concept of MPC's one directly finds a quantitative explanation in terms of the special kind of the arrangement of such mPC's in a paracrystalline superlattice (MPC). Here as well as in the atomic lattice of the MPC's nine new statistical parameters have to be introduced which describe the

Old concept microparacrystals

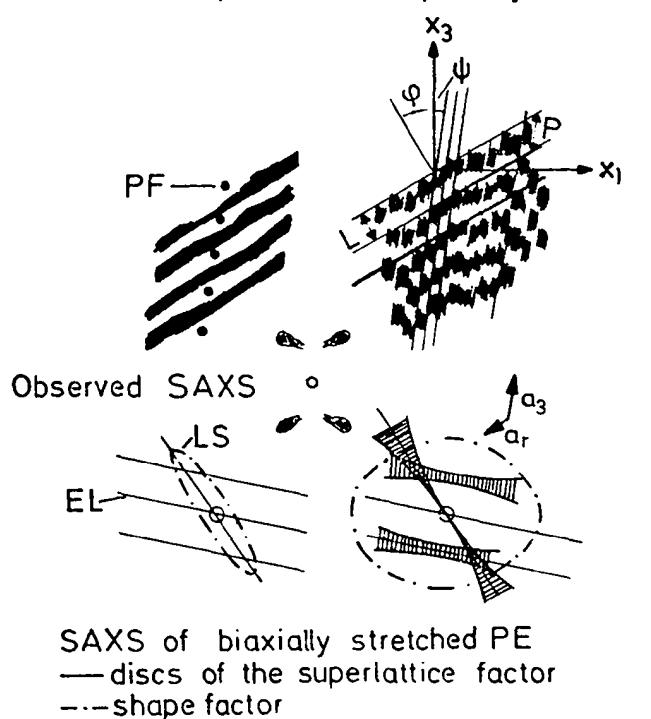

Fig. 1. The SAXS four-point diagram of biaxially oriented polyethylene. The conventional explanation is given on the left-hand side of the figure and the explanation in terms of mPC"s on the right-hand side of the figure. 
statistical (liquid-like) fluctuations of the edge vectors of the lattice cells. From the two-dimensional SAXS analysis one learns, for instance, that during annealing the vector $a_{r}$, which expands between the centers of laterally adjacent $\mathrm{mPC}$ 's, shows a decreasing fluctuation in chain direction 3 . The structure becomes bimodal after a certain annealing time indicating that groups of mPC's cluster together within the lamellae with well-aligned fold surfaces (Loboda-C̆ačković, Hosemann \& Čačković, 1971). From WAXS one learns that smaller mPC's recrystallize here to form larger ones.

The lateral grain boundaries of the MPC's proved by a combined SAXS and WAXS analysis play an important role in the understanding of the increase of the long period, $P$, with temperature. Surface premelting cannot explain this for polymers with a crystallinity below 0.5 (Yeh, Hosemann, Loboda-Čačković \& Čačković, 1976). The paracrystalline model gives a direct answer: mPC's with unprotected lateral boundaries (e.g. at the end of lamellae) offer an excess free energy. They dissolve by solid-state diffusion and parts of their segments crystallize to form parts of the adjacent two lamellae (Hosemann, 1962). A true melting occurs solely in temperature range III near the melting point where the SAXS intensity decreases and isolated groups of bounded bundles of lamellae are imbedded in the melt. This can be shown directly on isotactic poly(1-butene) which in this range only melts partially; quenched to room temperature it recrystallizes to the unstable modification II (Haase, Hosemann \& Renwanz, 1977). Even the so-called single crystals grown from dilute solution consist of specially aligned mPC's. Their degradation by $\mathrm{O}_{3}$ or $\mathrm{HNO}_{3}$ can only be understood in terms of the important role of the lateral grain boundaries of the mPC's (Schönfeld, Wilke, Höhne \& Hosemann, 1972; Hosemann, Cačković \& Loboda-Čačković, 1975).

The full paper will be published in Journal of Materials Science.

\section{References}

Haase, J., Hosemann, R. \& Renwanz, B. (1977). Colloid Polym. Sci. 255, 849-855.

HermanN, C. (1931a). Z. Kristallogr. 79, 186-221.

HermanN, C. (1931b). Z. Kristallogr. 79, 337--347.

Hosemann, R. (1962). Polymer, 3, 349-392.

hosemann, R., Cacković, H. \& Loboda-CaČ́ković, J. (1975). Makromol. Chem. 176, 3065-3077.

Kaji, K., Mochizuki, T., Akiyama, A. \& Hosemann, R. (1978). J. Mater. Sci. 13, 972-984.

Loboda-C̆ą̌ković, J., HosemanN, R. \& Čă̌ković, H. (1971). Kolloid Z. Z. Polym. 247, 824-829.

LOBODA-ČAČKOVIĆ, J., HOSEMANN, R., ČAČKOVIĆ, H., Ferrero, A. \& Ferracini, E. (1976). Polymer, 17, 303 308.

Noether, H. D. \& Whitney, W. (1973). Kolloid Z. Z. Polym. 251, 991-1005

SChÖNFEld, A., Wilke, W., HöHNe, G. \& HoSEMANN, R. (1972). Kolloid Z. Z. Polvm. 250, 102-110.

YeH, G. S. Y., HoSEMANN, R., LOBODA-ČAČKOVIĆ, J. \& C̆AČ́ović, H. (1976). Polymer, 17, 309-318.

J. Appl. Cryst. (1978). 11, 541-546

\title{
The Small-Angle Scattering of Distorted Lamellar Structures
}

\author{
By C. G. Vonk, DSM Central Laboratory, PO Box 18, Geleen, Netherlands
}

(Received 3 November 1977; accepted 31 May 1978)

\begin{abstract}
The effects of deviations from an ideal lamellar structure (infinite-size clusters of parallel layers of alternating electron densities) on the small-angle scattering curve are treated with the aid of the correlation function. If surrounded by a matrix of the average electron density, reduction of the size of the clusters in the direction of the layer normals leads to a simple modification of the one-dimensional correlation function. Distortions giving rise to structures containing concentric layers have little effect on this function, whereas corrugation of the surfaces causes minor modifications. Secondorder defects are shown to reduce the three-dimensional correlation function of the ideal structure $\gamma^{0}(r)$ according to $\gamma(r)=\gamma^{0}(r) \exp (-2 r / d)$, where $d$ is the 'distortion length'. This is the average length of the vectors for which the number of intersections with lamellar interfaces has changed by \pm 1 as a consequence of the distortions. Calculated diffraction curves show that the effects of reducing the cluster size and of increasing the width $\beta$ of the lamellar thickness distribution function are very similar. However, changes in $d$ and $\beta$ affect the scattering curves in a different way, which, other conditions being favourable, may enable these parameters to be determined from observed scattering curves.
\end{abstract}

\section{Introduction}

In nearly all calculations of the small-angle scattering of melt-crystallized polymers use is made of a structure model consisting of alternating parallel crystalline and amorphous layers in randomly oriented clusters, whose dimensions in all directions are large compared with those covered by the experiments. In the following this model will be referred to as the 'ideal lamellar model'. In the calculations two approaches have been followed; according to the first, and more general one, the one-dimensional intensity function $I_{1}$ from a cluster is obtained by squaring the corresponding one-dimensional amplitude function $F_{1}$, which in turn is obtained by Fourier transformation of the electron-density variations along the lamellar normal. A review of the literature about procedures and results, which also deals with the 